\title{
A VOLUME ESTIMATE FOR THE SET OF STABLE LATTICES
}

\author{
URI SHAPIRA AND BARAK WEISS
}

\begin{abstract}
We show that in high dimensions the set of stable lattices is almost of full measure in the space of unimodular lattices.
\end{abstract}

Let $G \stackrel{\text { def }}{=} \mathrm{SL}_{n}(\mathbb{R}), \Gamma \stackrel{\text { def }}{=} \mathrm{SL}_{n}(\mathbb{Z})$, and let $A \subset G$ denote the subgroup of diagonal matrices with positive entries. The quotient space $\mathcal{L}_{n} \stackrel{\text { def }}{=} G / \Gamma$ is naturally identified with the space of unimodular lattices in $\mathbb{R}^{n}$, and the group $G$ (and any of it subgroups) acts via left translations, or equivalently, by acting on lattices via its linear action on $\mathbb{R}^{n}$. A lattice $\Lambda$ is called stable if for any subgroup $\Delta \subset \Lambda$, one has $\operatorname{vol}(\Delta \otimes \mathbb{R} / \Delta) \geq 1$ (in the literature the term semi-stable is also used), and we denote the set of stable lattices by $\mathcal{S}^{(n)}$.

A central problem is to understand the orbits of the $A$-action on $\mathcal{L}_{n}$. In [SW] we proved that for any lattice $\Lambda \in \mathcal{L}_{n}$, the orbit-closure $\overline{A \Lambda}$ contains a stable lattice. This result reduces the proof of Minkowski's conjecture on the product of inhomogeneous linear forms to that of estimating the Euclidean covering radius of stable lattices (see [SW] for details). Understanding stable lattices is therefore a natural problem due to its connection both with well-studied problems in the geometry of numbers, and with dynamics of the $A$-action. Although $\mathcal{S}^{(n)}$ is compact (while $\mathcal{L}_{n}$ is not), in this note we show that $\mathcal{S}^{(n)}$ has almost full measure with respect to the natural probability measure on $\mathcal{L}_{n}$, for large $n$. Moreover the convergence to full measure is very fast. This answers a question we were asked by G. Harder, and can be viewed as a manifestation of the concentration of mass along the equator in high dimensional Euclidean balls.

We will prove the following.

Theorem 1. Let $m$ denote the $G$-invariant probability measure on $\mathcal{L}_{n}$ derived from Haar measure on $G$, and let $\mathcal{S}^{(n)}$ denote the subset of stable lattices in $\mathcal{L}_{n}$. Then there is a constant $C>0$ such that for all sufficiently large $n$,

$$
m\left(\mathcal{L}_{n} \backslash \mathcal{S}^{(n)}\right) \leq\left(\frac{C}{n}\right)^{\frac{n-1}{2}} .
$$

In particular $m\left(\mathcal{S}^{(n)}\right) \longrightarrow 1$ as $n \rightarrow \infty$. 
For $\Lambda \in \mathcal{L}_{n}$ and a subgroup $\Delta \subset \Lambda$, we denote by $r(\Delta)$ its rank and by $|\Delta|$ its covolume in the Euclidean subspace $\Delta \otimes \mathbb{R} \subset \mathbb{R}^{n}$. For $k=1, \ldots, n-1$ let us denote $\mathcal{V}_{k}(\Lambda) \stackrel{\text { def }}{=}\left\{|\Delta|^{1 / k}: \Delta \subset \Lambda, r(\Delta)=k\right\}$ and $\alpha_{k}(\Lambda)=\min \mathcal{V}_{k}(\Lambda)$ so that $\Lambda$ is stable if and only if $\alpha_{k}(\Lambda) \geq 1$ for $k=1, \ldots, n-1$. Let

$$
\mathcal{S}_{k}^{(n)}(t) \stackrel{\text { def }}{=}\left\{x \in \mathcal{L}_{n}: \alpha_{k}(x) \geq t\right\}, \quad \mathcal{S}_{k}^{(n)} \stackrel{\text { def }}{=} \mathcal{S}_{k}^{(n)}(1) .
$$

With this notation $\mathcal{S}^{(n)}=\bigcap_{k=1}^{n-1} \mathcal{S}_{k}^{(n)}$. We will show:

Proposition 2. There is $C>0$ such that for all sufficiently large $n$, and all $k \in\{1, \ldots, n-1\}$,

$$
m\left(\mathcal{L}_{n} \backslash \mathcal{S}_{k}^{(n)}\right) \leq \frac{1}{n}\left(\frac{C}{n}\right)^{\frac{k(n-k)}{2}} .
$$

Proof of Theorem 1. For $n>C$, the largest value of $\left(\frac{C}{n}\right)^{\frac{k(n-k)}{2}}$ is attained when $k=1$ and $k=n-1$. Therefore (1) implies

$$
\begin{aligned}
m\left(\mathcal{L}_{n} \backslash \mathcal{S}^{(n)}\right) & =m\left(\mathcal{L}_{n} \backslash \bigcap_{k=1}^{n-1} \mathcal{S}_{k}^{(n)}\right)=m\left(\bigcup_{k=1}^{n-1} \mathcal{L}_{n} \backslash \mathcal{S}_{k}^{(n)}\right) \\
& \leq \frac{n-2}{n}\left(\frac{C}{n}\right)^{\frac{n-1}{2}} \leq\left(\frac{C}{n}\right)^{\frac{n-1}{2}} .
\end{aligned}
$$

We will also show:

Proposition 3. There is $C_{1}>0$ such that if we set

$$
t_{k}=t(n, k) \stackrel{\text { def }}{=}\left(\frac{n}{C_{1}}\right)^{\frac{n-k}{2 n}}
$$

then

$$
\max _{k=1, \ldots, n-1} m\left(\mathcal{L}_{n} \backslash \mathcal{S}_{k}^{(n)}\left(t_{k}\right)\right)=o\left(\frac{1}{n}\right) .
$$

In particular, $m\left(\bigcap_{k=1}^{n-1} \mathcal{S}_{k}^{(n)}\left(t_{k}\right)\right) \rightarrow_{n \rightarrow \infty} 1$.

Remarks. 1. Let us define $\bar{\alpha}_{n, k} \stackrel{\text { def }}{=} \sup \left\{\alpha_{k}(\Lambda): \Lambda \in \mathcal{L}_{n}\right\}$. These quantities are powers of the so-called Rankin constants or generalized Hermite constants usually denoted by $\gamma_{n, k}$ (see [Thu98]), namely they are related by

$$
\bar{\alpha}_{n, k}^{2 k}=\gamma_{n, k} \text {. }
$$

The origin of this exponent $2 k$ is the $1 / k$ in the definition of $\mathcal{V}_{k}$, which we have imposed so that the functions $\alpha_{k}$ behave nicely with respect to homothety. This normalization has the additional advantage that the growth rate of the different $\bar{\alpha}_{n, k}$ (as a function of $n$ ) becomes the same for all $k$. Namely [Thu98, Cor. 2] and (3) show that $\log \bar{\alpha}_{n, k}=\frac{1}{2} \log n+O(1)$ (where the implicit constant depends on $k$ ). 
2. It seems plausible that most lattices come close to realizing the Rankin constants, that is, for any $\varepsilon>0$,

$$
\lim _{n \rightarrow \infty} m\left(\left\{\Lambda \in \mathcal{L}_{n}: \forall k, \alpha_{k}(\Lambda)>\bar{\alpha}_{n, k}-\varepsilon\right\}\right)=1 .
$$

Combined with the result of Thunder mentioned above, Proposition 3 may be viewed as supporting evidence for such a conjecture.

3. We take this opportunity to formulate an analogous question regarding the covering radius; that is, is it true that for any $\varepsilon>0$,

$$
\lim _{n \rightarrow \infty} m\left\{\Lambda \in \mathcal{L}_{n}: \operatorname{covrad}(\Lambda)<\inf _{\Lambda^{\prime} \in \mathcal{L}_{n}} \operatorname{covrad}\left(\Lambda^{\prime}\right)+\varepsilon\right\}=1,
$$

where

$$
\operatorname{covrad}(\Lambda)=\inf \left\{r>0: \mathbb{R}^{n}=\Lambda+B(0, r)\right\}
$$

and $B(0, r)$ is the Euclidean ball of radius $r$ around the origin.

The proof of Propositions 2 and 3 relies on Thunder's work and on a variant of Siegel's formula [Sie45] which relates the Lebesgue measure on $\mathbb{R}^{n}$ and the measure $m$ on $\mathcal{L}_{n}$. We now review Siegel's method and Thunder's results.

In the sequel we consider $n \geq 2$ and $k \in\{1, \ldots, n-1\}$ as fixed and omit, unless there is risk of confusion, the symbols $n$ and $k$ from the notation. Consider the (set valued) map $\Phi=\Phi_{k}^{(n)}$ that assigns to each lattice $\Lambda \in \mathcal{L}_{n}$ the following subset of the exterior power of $\bigwedge^{k} \mathbb{R}^{n}$ :

$$
\Phi(\Lambda) \stackrel{\text { def }}{=}\left\{ \pm w_{\Delta}: \Delta \subset \Lambda \text { a primitive subgroup with } r(\Delta)=k\right\}
$$

where $w_{\Delta} \stackrel{\text { def }}{=} v_{1} \wedge \cdots \wedge v_{k}$ and $\left\{v_{i}\right\}_{i=1}^{k}$ is a basis for $\Delta$ (note that $w_{\Delta}$ is well-defined up to sign, and $\Phi(\Lambda)$ contains both possible choices). Let

$$
\mathscr{V}=\mathscr{V}_{k}^{(n)} \stackrel{\text { def }}{=}\left\{v_{1} \wedge \cdots \wedge v_{k}: v_{i} \in \mathbb{R}^{n}\right\} \backslash\{0\}
$$

be the variety of pure tensors in $\bigwedge^{k} \mathbb{R}^{n}$. For any compactly supported bounded Riemann integrable ${ }^{1}$ function $f$ on $\mathscr{V}$ set

$$
\hat{f}: \mathcal{L}_{n} \rightarrow \mathbb{R}, \quad \hat{f}(\Lambda) \stackrel{\text { def }}{=} \sum_{w \in \Phi(\Lambda)} f(w) .
$$

Then it is known (see [Wei82, Lemma 2.4.2]) that the (finite) sum (4) defines a function in $L^{1}\left(\mathcal{L}_{n}, m\right)$. This allows us to define a Radon measure $\theta=\theta_{k}^{(n)}$ on $\mathscr{V}$ by the formula

$$
\int_{\mathscr{V}} f d \theta \stackrel{\text { def }}{=} \int_{\mathcal{L}_{n}} \hat{f} d m, \text { for } f \in C_{c}(\mathscr{V}) .
$$

Write $G=G_{n} \stackrel{\text { def }}{=} \mathrm{SL}_{n}(\mathbb{R})$. There is a natural transitive action of $G_{n}$ on $\mathscr{V}$ and the stabilizer of $e_{1} \wedge \cdots \wedge e_{k}$ is the subgroup

$$
H=H_{k}^{(n)} \stackrel{\text { def }}{=}\left\{\left(\begin{array}{cc}
A & B \\
0 & D
\end{array}\right) \in G: A \in G_{k}, D \in G_{n-k}\right\} .
$$

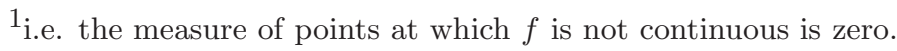


We therefore obtain an identification $\mathscr{V} \simeq G / H$ and view $\theta$ as a measure on $G / H$. It is well-known (see e.g. [Wei82]) that up to a proportionality constant there exists a unique $G$-invariant measure $m_{G / H}$ on $G / H$; moreover, given Haar measures $m_{G}, m_{H}$ on $G$ and $H$ respectively, there is a unique normalization of $m_{G / H}$ such that for any $f \in L^{1}\left(G, m_{G}\right)$

$$
\int_{G} f d m_{G}=\int_{G / H} \int_{H} f(g h) d m_{H}(h) d m_{G / H}(g H) .
$$

We choose the Haar measure $m_{G}$ so that it descends to our probability measure $m$ on $\mathcal{L}_{n}$; similarly, we choose the Haar measure $m_{H}$ so that the periodic orbit $H \mathbb{Z}^{n} \subset \mathcal{L}_{n}$ has volume 1. These choices of Haar measures determine our measure $m_{G / H}$ unequivocally. It is clear from the defining formula (5) that $\theta$ is $G$-invariant and therefore the two measures $m_{G / H}, \theta$ are proportional. In fact (see [Sie45] for the case $k=1$ and [Wei82, Lemma 2.4.2] for the general case),

$$
m_{G / H}=\theta \text {. }
$$

For $t>0$, let $\chi=\chi_{t}: \mathscr{V} \rightarrow \mathbb{R}$ be the restriction to $\mathscr{V}$ of the characteristic function of the ball of radius $t$ around the origin, in $\bigwedge^{k} \mathbb{R}^{n}$, with respect to the natural inner product obtained from the Euclidean inner product on $\mathbb{R}^{n}$. Note that $\hat{\chi}(x)=0$ if and only if $x \in \mathcal{S}_{k}^{(n)}\left(t^{1 / k}\right)$ and furthermore, $\hat{\chi}(x) \geq 1$ if $x \in \mathcal{L}_{n} \backslash \mathcal{S}_{k}^{(n)}\left(t^{1 / k}\right)$. It follows that

$$
m\left(\mathcal{L}_{n} \backslash \mathcal{S}_{k}^{(n)}(t)\right) \leq \int_{\mathcal{L}_{n}} \widehat{\left(\chi_{t^{k}}\right)} d m=\int_{\mathscr{V}} \chi_{t^{k}} d \theta .
$$

Let $V_{j}$ denote the volume of the Euclidean unit ball in $\mathbb{R}^{j}$ and let $\zeta$ denote the Riemann zeta function. We will use an unconventional convention $\zeta(1)=1$, which will make our formulae simpler. For $j \geq 1$, define

$$
R(j) \stackrel{\text { def }}{=} \frac{j^{2} V_{j}}{\zeta(j)} \text { and } B(n, k) \stackrel{\text { def }}{=} \frac{\prod_{j=1}^{n} R(j)}{\prod_{j=1}^{k} R(j) \prod_{j=1}^{n-k} R(j)} .
$$

The following is [Thu98, Lemma 5]:

Theorem 4 (Thunder). For $t>0$, we have $\int_{\mathscr{V}} \chi_{t} d m_{G / H}=B(n, k) \frac{t^{n}}{n}$.

(Note that in Thunder's notation, by [Thu98, $\S 4], c(n, k)=B(n, k) / n$.)

We will need to bound $B(n, k)$.

Lemma 5. There is $C>0$ so that for all large enough $n$ and all $k=$ $1, \ldots, n-1$,

$$
B(n, k) \leq\left(\frac{C}{n}\right)^{\frac{k(n-k)}{2}}
$$


Proof. In this proof $c_{0}, c_{1}, \ldots$ are constants independent of $n, k, j$. Because of the symmetry $B(n, k)=B(n, n-k)$ it is enough to prove (9) with $k \leq \frac{n}{2}$. Using the formula $V_{j}=\frac{\pi^{j / 2}}{\Gamma\left(\frac{j}{2}+1\right)}$ we obtain

$$
\begin{aligned}
B(n, k) & =\prod_{j=1}^{k} \frac{R(n-k+j)}{R(j)}=\prod_{j=1}^{k} \frac{\zeta(j)(n-k+j)^{2} \frac{\pi^{(n-k+j) / 2}}{\Gamma\left(\frac{n-k+j}{2}+1\right)}}{\zeta(n-k+j) j^{2} \frac{\pi^{j / 2}}{\Gamma\left(\frac{j}{2}+1\right)}} \\
& =\prod_{j=1}^{k} \frac{\zeta(j)}{\zeta(n-k+j)} \cdot\left(\frac{n-k+j}{j}\right)^{2} \cdot \pi^{\frac{n-k}{2}} \cdot \frac{\Gamma\left(\frac{j}{2}+1\right)}{\Gamma\left(\frac{n-k+j}{2}+1\right)} .
\end{aligned}
$$

Note that $\zeta(s) \geq 1$ is a decreasing function of $s>1$, so (recalling our convention $\zeta(1)=1) \frac{\zeta(j)}{\zeta(n-k+j)} \leq c_{0} \stackrel{\text { def }}{=} \zeta(2)$. It follows that for all large enough $n$ and for any $1 \leq j \leq k$,

$$
\frac{\zeta(j)}{\zeta(n-k+j)} \cdot\left(\frac{n-k+j}{j}\right)^{2} \cdot \pi^{\frac{n-k}{2}} \leq c_{0} n^{2} \pi^{\frac{n-k}{2}} \leq 4^{\frac{n-k}{2}}
$$

According to Stirling's formula, there are positive constants $c_{1}, c_{2}$ such that for all $x \geq 2$,

$$
c_{1} \sqrt{\frac{2 \pi}{x}}\left(\frac{x}{e}\right)^{x} \leq \Gamma(x) \leq c_{2} \sqrt{\frac{2 \pi}{x}}\left(\frac{x}{e}\right)^{x} .
$$

We set $u \stackrel{\text { def }}{=} \frac{j}{2}+1$ and $v \stackrel{\text { def }}{=} \frac{n-k}{2}$, so that $u+v \geq \frac{n-1}{4}$, and obtain

$$
\begin{aligned}
\frac{\Gamma\left(\frac{j}{2}+1\right)}{\Gamma\left(\frac{n-k+j}{2}+1\right)} & =\frac{\Gamma(u)}{\Gamma(u+v)} \leq \frac{c_{2}}{c_{1}} \sqrt{\frac{u+v}{u}} \frac{u^{u}}{(u+v)^{u+v}} \frac{e^{u+v}}{e^{u}} \\
& \leq c_{3} e^{v} \frac{u^{u-1 / 2}}{(u+v)^{u+v-1 / 2}}=c_{3}\left(\frac{e}{u+v}\right)^{v} \frac{1}{\left(1+\frac{v}{u}\right)^{u-1 / 2}} \\
& \leq c_{3}\left(\frac{4 e}{n-1}\right)^{\frac{n-k}{2}} .
\end{aligned}
$$

Using (10) and (11) we obtain

$$
B(n, k) \leq\left[c_{3} 4^{\frac{n-k}{2}}\left(\frac{4 e}{n-1}\right)^{\frac{n-k}{2}}\right]^{k}=\left[c_{3}\left(\frac{16 e}{n-1}\right)^{\frac{n-k}{2}}\right]^{k} .
$$

So taking $C>16 c_{3} e$ we obtain (9) for all large enough $n$.

Proof of Propositions 2 and 3. Let $C$ be as in Lemma 5 and let $C_{1}>C$. For Proposition 3, using (8), (7) and Theorem 4, for all sufficiently large $n$ we have

$$
\begin{aligned}
m\left(\mathcal{L}_{n} \backslash \mathcal{S}_{k}^{(n)}\left(t_{k}\right)\right) & \leq B(n, k) \frac{t_{k}^{k n}}{n} \\
& \leq \frac{1}{n}\left(\frac{C}{n}\right)^{\frac{k(n-k)}{2}}\left(\frac{n}{C_{1}}\right)^{\frac{k(n-k)}{2}}=\frac{1}{n}\left(\frac{C}{C_{1}}\right)^{\frac{k(n-k)}{2}} .
\end{aligned}
$$


Multiplying by $n$ and taking the maximum over $k$ we obtain

$$
n \max _{k=1, \ldots, n} m\left(\mathcal{L}_{n} \backslash \mathcal{S}_{k}^{(n)}\left(t_{k}\right)\right) \leq\left(\frac{C}{C_{1}}\right)^{\frac{n-1}{2}} \rightarrow_{n \rightarrow \infty} 0 .
$$

The proof of Proposition 2 is identical using $t=1$ instead of $t_{k}$.

Acknowledgements. The authors thank Prof. Günter Harder for an interesting conversation regarding stable lattices and for raising the question addressed in this article. The work of the authors was supported by ERC starter grant DLGAPS 279893 and ISF grants 190/08, 357/13, and the Chaya Fellowship. The results of this paper appeared on arXiv as part of a preliminary version of [SW].

\section{REFERENCES}

[Sie45] C. L. Siegel, A mean value theorem in geometry of numbers, Ann. of Math. (2) 46 (1945), 340-347. MR0012093 (6,257b)

[SW] U. Shapira and B. Weiss, Stable lattices and the diagonal group. To appear in JEMS.

[Thu98] J. L. Thunder, Higher-dimensional analogs of hermite's constant, The Michigan Mathematical Journal 45 (1998), no. 2, 301-314.

[Wei82] A. Weil, Adeles and algebraic groups, Progress in Mathematics, vol. 23, Birkhäuser Boston, Mass., 1982. With appendices by M. Demazure and Takashi Ono. MR670072 (83m:10032)

Dept. of Mathematics, Technion, Haifa, IsRael ushapira@tx.technion.ac.il

Dept. of Mathematics, Tel Aviv University, Tel Aviv, IsRael barakw@post.tau.ac.il 